\title{
New Developments in Urology: Urology Update 2014
}

\author{
Sender Herschorn, MD, FRCSC
}

Division of Urology, University of Toronto, Sunnybrook Health Sciences Centre, Toronto, ON

Cite as: Can Urol Assoc J 2015;9(1-2):S1-2. http://dx.doi.org/10.5489/cuaj.2668 Published online February 9, 2015.

$\mathrm{T}$ he University of Toronto Urology Update has been held annually since 1993 to give practicing urologists and urology residents and fellows a review of current knowledge, presentations of new ideas, and interpretations of controversies to help facilitate management of clinical problems. This year was no exception, with many new developments in diverse areas of urology covered at the update.

Prostate cancer ( $\mathrm{PCa})$ screening has been particularly controversial, as many Canadian urologists, individually and collectively through the Canadian Urological Association (CUA), voiced their concerns over the latest recommendations of the Canadian Task Force on Preventive Health Care (CTFPHC). Apart from Dr. Anthony Miller, who defended the recommendations against prostate-specific antigen (PSA) screening, the other speakers presented information that calls into question the process and conclusions of the task force.

Dr. H. Ballentine Carter underscored the importance of detecting and treating high-risk PCa; this led into a session on the use of magnetic resonance imaging (MRI) for PCa diagnosis and staging. MRI is currently being evaluated as a screening tool, and preliminary evidence indicates that it may identify clinically significant cancers. It has also been reported to be useful in directing ultrasound (US)-guided biopsies to specific regions of the prostate, with the use of an image fusion device that fuses MRI data with real-time US imaging.

Factors associated with PCa incidence and progression were discussed. Diet-induced hyperinsulinemia has been experimentally shown to be associated with accelerated growth of PCa cell (LNCaP) xenografts. The commonly used antidiabetic drug, metformin, may influence cancer cells indirectly by decreasing insulin levels or directly by influencing cancer cell proliferation and apoptosis. ${ }^{1}$ New data were presented from a single-armed pilot study on the neoadjuvant use of metformin prior to radical prostatectomy. Other factors in the disease may be male-pattern baldness and prostate inflammation.

Dr. Anthony Burnett reviewed penile rehabilitation following nerve-sparing radical prostatectomy. Multiple interventions may be effective; however, the use of daily PDE5 therapy has not consistently been shown to be more beneficial than ondemand treatment.

Dr. Anne Gormley provided an overview of the latest guidelines on overactive bladder $(\mathrm{OAB})$ from the American Urological Association/Society of Urodynamics, Female Pelvic Medicine and Urogenital Reconstruction (AUA/SUFU). First-line treatment includes behavioural therapy alone or combined with pharmacologic therapy. Second-line therapy includes oral or cutaneous forms of antimuscarinics and, because of recent evidence, a $\beta 3$-adrenergic agonist. The refractory patient was specifically defined and that patient may be offered evaluation by an appropriate specialist if he/she desires additional therapy. Accumulating evidence has confirmed the value of third-line therapy with intradetrusor injections of onabotulinumtoxinA and neuromodulation. Neuromodulation can be non-invasive with percutaneous tibial nerve stimulation (PTNS) or sacral nerve stimulation (SNS) for carefully selected patients with severe refractory $O A B$ who are willing to undergo a surgical procedure. Although PTNS is the least costly option in the United States, the lack of coverage for this modality in the Canadian health-care system probably makes it the least used form of neuromodulation in this country.

The major benefit of mirabegron is its lack of antimuscarinic side effects. There are Phase 2 data indicating a benefit of combining mirabegron and solifenacin, and a large Phase 3 study is underway to test the benefit of the combination. Since OAB causation is multifactorial, drug combinations make sense. In nocturia, a new low-dose oral desmopressin tablet (DDAVP) may allow more patients with nocturnal polyuria to be treated.

New data on postoperative percutaneous nephrolithomy drainage may stimulate us to look at alternatives to large-calibre catheter drainage. These include using a smaller tube or not using a tube at all in selected patients.

Dr. Mark Cain presented his large experience of pediatric augmentation cystoplasty. He reviewed the various problems that may be encountered, and highlighted the need for organized transition of these and other pediatric patients into the adult environment.

The following pages summarize some of the highlights from material presented on those topics during the two-day sympo- 
sium. Please join us next year for Urology Update 2015, on October 30-31.

\section{Reference}

1. Pollak M. Metformin and other biguanides in oncology: Advancing the research agenda. Can Prev Res 2010;3:1060-5.
Competing interests: Dr. Herschorn is a member of advisory boards for, and has received payment and grants from, Allergan, Astellas, Ferring, Lilly, Merus, and Pfizer. He is currently participating in clinical trials with Allergan, Astellas, and Cook.

Correspondence: Dr. Sender Herschorn, Division of Urology, University of Toronto, Sunnybrook Health Sciences Centre, Rm. A309, 2075 Bayview Ave., Toronto 0N M4N 3M5; s.herschorn@utoronto.ca 